\title{
On the General Randić index of polymeric networks modelled by generalized Sierpiński graphs
}

\author{
Alejandro Estrada-Moreno and Juan A. Rodríguez-Velázquez \\ Departament d'Enginyeria Informàtica i Matemàtiques, \\ Universitat Rovira i Virgili, Av. Països Catalans 26, 43007 Tarragona, Spain. \\ alejandro.estrada@urv.cat,juanalberto.rodriguez@urv.cat
}

March 2, 2022

\begin{abstract}
The General Randić index $R_{\alpha}$ of a simple graph $G$ is defined as

$$
R_{\alpha}(G)=\sum_{v_{i} \sim v_{j}}\left(d\left(v_{i}\right) d\left(v_{j}\right)\right)^{\alpha}
$$

where $d\left(v_{i}\right)$ denotes the degree of the vertex $v_{i}$. Rodríguez-Velázquez and Tomás-Andreu [MATCH Commun. Math. Comput. Chem. 74 (1) (2015) 145160] obtained closed formulae for the Randić index $R_{-1 / 2}$ of Sierpiński-type polymeric networks, where the base graph is a complete graph, a triangle-free regular graph or a bipartite semiregular graph. In the present article we obtain closed formulae for the general Randić index $R_{\alpha}$ of Sierpinski-type polymeric networks, where the base graph is arbitrary.
\end{abstract}

\section{Introduction}

Around the middle of the last century theoretical chemists proposed the use of topological indices to obtain information on the dependence of various properties of organic substances on molecular structure. In this sense, a large number of various topological indices was proposed and considered in the chemical literature [36]. We highlight the article [2] where Camarda and Maranas addressed the design of polymers with optimal levels of macroscopic properties through the use of topological indices. Specifically, in the above mentioned article two zeroth-order and two first-order connectivity indices were employed for the first time as descriptors in structure-property correlations in an optimization study. Based on these descriptors, a set of new correlations for heat capacity, cohesive energy, glass transition temperature, refractive index, and dielectric constant were proposed. 
The molecular structure-descriptor, introduced in 1975 by Milan Randić in [29], is defined as

$$
R(G)=\sum_{v_{i} v_{j} \in E} \frac{1}{\sqrt{d\left(v_{i}\right) d\left(v_{j}\right)}},
$$

where $d\left(v_{i}\right)$ represents the degree of the vertex $v_{i}$ in $G=(V, E)$. Nowadays, $R(G)$ is referred to as the Randić index of $G$. This graph topological index, sometimes referred to as connectivity index, has been successfully related to a variety of physical, chemical, and pharmacological properties of organic molecules and became one of the most popular molecular-structure descriptors [30]. After the publication of the first paper [29], mathematical properties and generalizations of $R(G)$ were extensively studied, for instance, see $[6,10,15,22-26,31,32,34,38]$ and the references cited therein. The Randic index was generalized by Gutman and Lepović in [11] as

$$
R_{\alpha}(G)=\sum_{v_{i} v_{j} \in E}\left(d\left(v_{i}\right) d\left(v_{j}\right)\right)^{\alpha}, \alpha \neq 0
$$

Obviously, the standard Randić index is obtained when $\alpha=-1 / 2$. In the chemical literature the quantity

$$
R_{1}(G)=\sum_{v_{i} v_{j} \in E} d\left(v_{i}\right) d\left(v_{j}\right)
$$

is called the second Zagreb index [4].

Some topological indices have been studied also for the case of polymeric networks. For instance, we cite the article [37], where the authors gave the explicitly formula of the $k$-connectivity index of an infinite class of dendrimer nanostars.

Over the past three decades, polymer networks has emerged as a coherent subject area $[1,16,17,34,35]$. While the basic works on polymer modelling started from linear polymeric systems, in recent years the attention has focused more and more on complex underlying geometries including fractal-type networks. It is well-known that, in comparison with those linear polymers, the properties of polymer networks depend to a much larger extent on methods and condition of preparation, i.e., properties depend not only on the chemical structure of the individual polymer chains, but on how those chains are joined together to form a network [35]. In this article we consider a model of polymer networks based on generalized Sierpiński graphs, which was previously studied in [34].

To begin with, we need some notation and terminology. Let $G=(V, E)$ be a nonempty graph of order $n$ and vertex set $V=\{1,2, \ldots, n\}$. We denote by $\{1,2, \ldots, n\}^{t}$ the set of words of length $t$ on alphabet $\{1,2, \ldots, n\}$. The letters of a word $u$ of length $t$ are denoted by $u_{1} u_{2} \cdots u_{t}$. The concatenation of two words $u$ and $v$ is denoted by $u v$. Klavžar and Milutinović introduced in [18] the graph $S\left(K_{n}, t\right)$ whose vertex set is $\{1,2, \ldots, n\}^{t}$, where $\{u, v\}$ is an edge if and only if there exists $i \in\{1, \ldots, t\}$ such that:

$$
\text { (i) } u_{j}=v_{j} \text {, if } j<i \text {; (ii) } u_{i} \neq v_{i} \text {; (iii) } u_{j}=v_{i} \text { and } v_{j}=u_{i} \text { if } j>i \text {. }
$$

The graph $S\left(K_{3}, t\right)$ is isomorphic to the graph of the Tower of Hanoi with $t$ disks [18]. Later, those graphs have been called Sierpiński graphs in [19] and they 
were studied by now from numerous points of view. The reader is invited to read, for instance, the following recent papers $[8,12-14,19-21,27]$ and references therein.

This construction was generalized in [9] for any graph $G=(V, E)$, by defining the generalized Sierpinski graph, $S(G, t)$, as the graph with vertex set $\{1,2, \ldots, n\}^{t}$ and edge set defined as follows. $\{u, v\}$ is an edge if and only if there exists $i \in\{1, \ldots, t\}$ such that:

(i) $u_{j}=v_{j}$, if $j<i$; (ii) $u_{i} \neq v_{i}$ and $\left\{u_{i}, v_{i}\right\} \in E$; (iii) $u_{j}=v_{i}$ and $v_{j}=u_{i}$ if $j>i$.

Notice that if $\{u, v\}$ is an edge of $S(G, t)$, there is an edge $\{x, y\}$ of $G$ and a word $w$ such that $u=w x y y \cdots y$ and $v=w y x x \cdots x$. In general, $S(G, t)$ can be constructed recursively from $G$ with the following process: $S(G, 1)=G$ and, for $t \geq 2$, we copy $n$ times $S(G, t-1)$ and add the letter $x$ at the beginning of each label of the vertices belonging to the copy of $S(G, t-1)$ corresponding to $x$. Then for every edge $\{x, y\}$ of $G$, add an edge between vertex $x y y \cdots y$ and vertex $y x x \cdots x$. See, for instance, Figure 1 that shows a graph $G$ and the generalized Sierpiński graph $S(G, 2)$. Besides Figure 2 shows the generalized Sierpiński graph $S(G, 3)$. Vertices of the form $x x \cdots x$ are called extreme vertices. Notice that for any graph $G$ of order $n$ and any integer $t \geq 2, S(G, t)$ has $n$ extreme vertices and, if $x$ has degree $d(x)$ in $G$, then the extreme vertex $x x \cdots x$ of $S(G, t)$ also has degree $d(x)$. Moreover, the degrees of two vertices $y x x \cdots x$ and $x y y \cdots y$, which connect two copies of $S(G, t-1)$, are equal to $d(x)+1$ and $d(y)+1$, respectively.
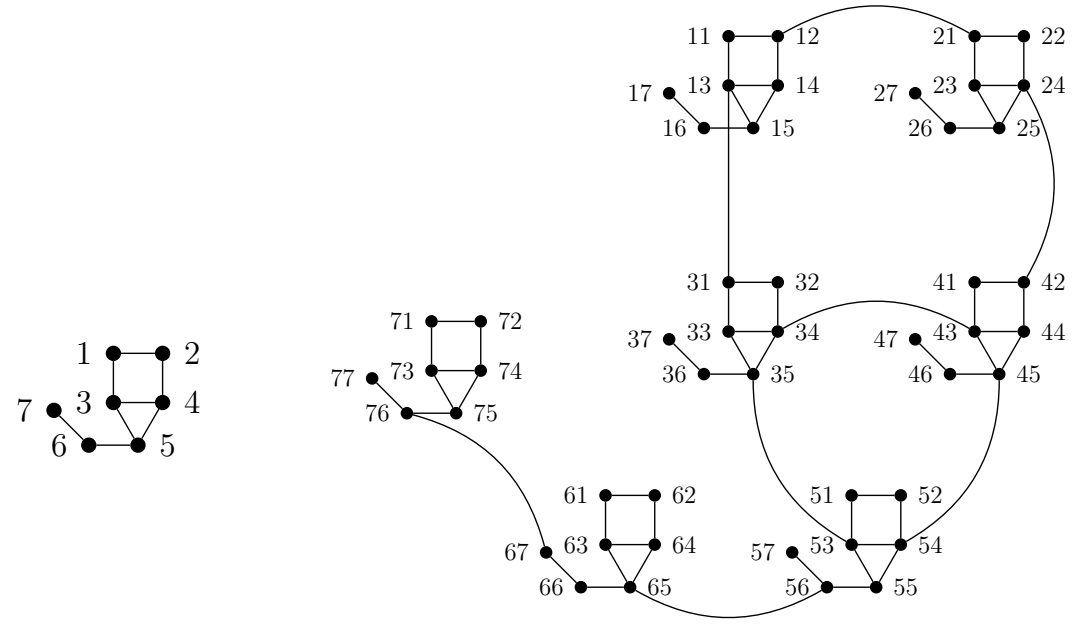

Figure 1: A graph $G$ and the Sierpiński graph $S(G, 2)$.

We denote by $P_{r}$ the path graph of order $r$. Notice that for $G=K_{2}$ we obtain $S\left(K_{2}, 2\right)=P_{4}$ and, in general, $S\left(K_{2}, t\right)=P_{2^{t}}$, which is the simplest possible polymer model presented by the ideal chain. Also, the graphs $S\left(K_{n}, t\right)$ were used in $[1,16,17]$ to analyse the scaling behaviour of experimentally accessible dynamical relaxation forms for polymers modelled through finite Sierpiński-type graphs, which we denote here by $P\left(K_{n}, t\right)$. Using the approach developed in $[16,17]$ to construct $P\left(K_{n}, t\right)$, now we define the polymeric Sierpiński graphs $P(G, t)=\left(V^{*}, E^{*}\right)$ introduced in [34], 


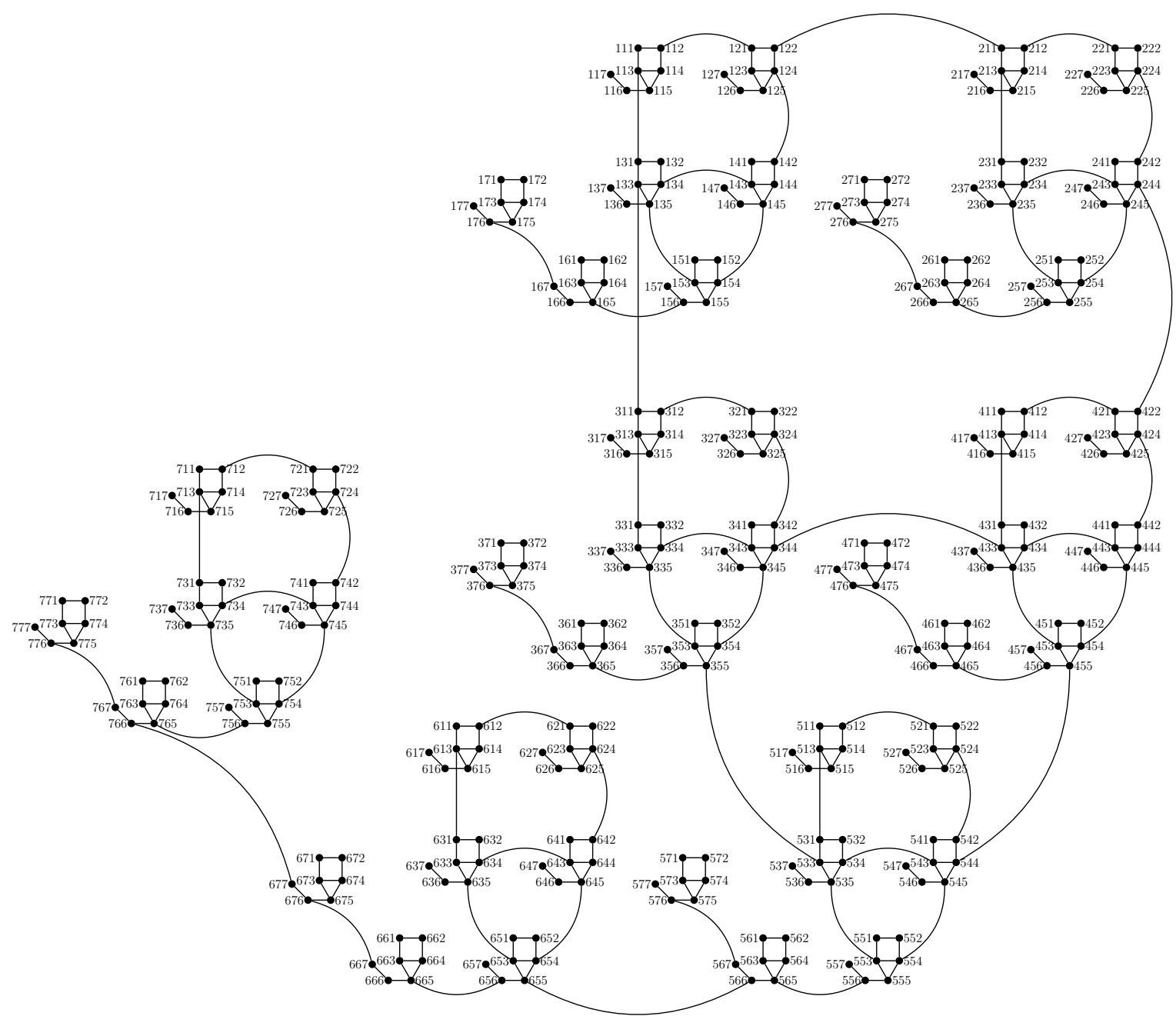

Figure 2: The Sierpiński graph $S(G, 3)$ for the graph $G$ of Figure 1.

where $G$ is a connected graph of order $n$ and $t$ is a positive integer. For $i \in\{1, \ldots, t\}$ we define the sets $A_{i}=\left\{a_{i_{1}}, \ldots, a_{i_{n i-1}}\right\}$ and we denote $S(G, i)=\left(V_{i}, E_{i}\right)$ and $V_{i}=$ $\left\{v_{i_{1}}, \ldots, v_{i_{n}}\right\}$. Then, the vertex set of $P(G, t)$ is

$$
V^{*}=\bigcup_{i=1}^{t}\left(A_{i} \cup V_{i}\right)
$$

and the edge set of $P(G, t)$ is

$$
E^{*}=\left(\bigcup_{i=1}^{n}\left(E_{i} \cup B_{i}\right)\right) \cup\left(\bigcup_{i=1}^{t-1} C_{i}\right),
$$

where $C_{i}=\left\{\left\{v_{i_{j}}, a_{(i+1)_{j}}\right\}: j=1, \ldots, n^{i}\right\}, B_{i}=\bigcup_{j=1}^{n^{i-1}} W_{j}$, and $W_{j}$ is formed by the edges obtained by connecting $a_{i_{j}}$ to every vertex belonging to the $j$-th copy of $G$ in $S(G, i)$. In other words, we construct $P(G, t)$ as follows: The iterative construction 
starts from one vertex, $a_{1_{1}}$, and one copy of $G=S(G, 1)$. So, we obtain $P(G, 1)$ by connecting $a_{1_{1}}$ to every vertex of $S(G, 1)$. To obtain $P(G, 2)$ we take $P(G, 1)$, $A_{2}$ and $S(G, 2)$. Then we connect each element $a_{2_{j}} \in A_{2}$ to $v_{1_{j}} \in V_{1}$ and we also connect $a_{2 j}$ to every vertex in the $j$-th copy of $G$ in $S(G, 2)$. Analogously, for the construction of $P(G, t)$ we take $P(G, t-1), A_{t}$ and $S(G, t)$. Then, we connect each element $a_{t_{j}} \in A_{t}$ to $v_{t-1_{j}} \in V_{t-1}$ and we also connect $a_{t_{j}}$ to every vertex in the $j$-th copy of $G$ in $S(G, t)$. Notice that $P\left(K_{3}, 2\right)=S\left(K_{4}, 2\right), S\left(K_{3}, 2\right)=P\left(K_{2}, 2\right)$, while for $t \geq 3, P\left(K_{n}, t\right) \neq S\left(K_{n+1}, t\right)$. Figure 3 shows a sketch of a polymeric Sierpiński graph $P(G, 2)$.

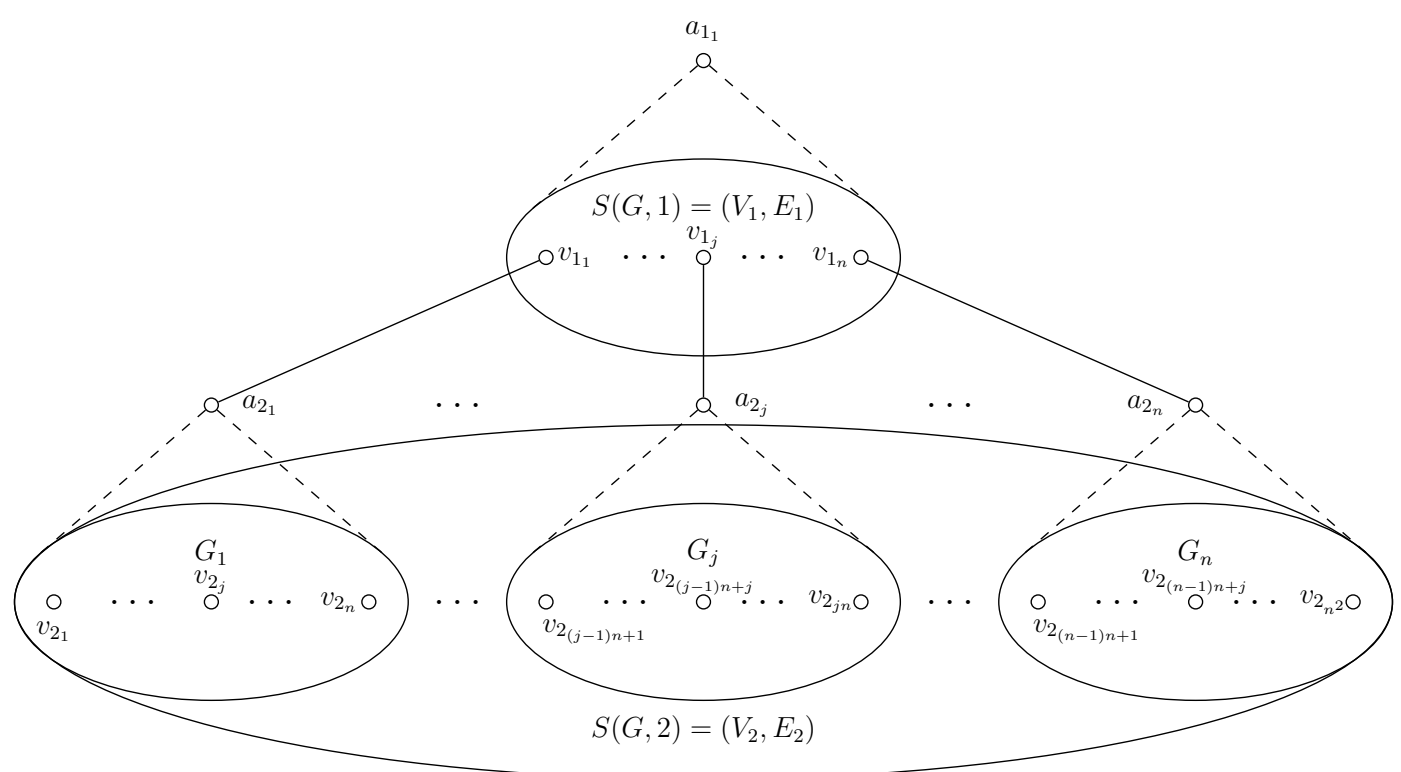

Figure 3: Sketch of a polymeric Sierpiński graph $P(G, 2)$, where a small ellipse represents a copy of $G$ and the dashed lines connecting a vertex $a_{i_{k}}$ and a small ellipse mean that each vertex of the $k$-th copy of $G$ in $S(G, 2)$ is connected to $a_{i_{k}}$.

The authors of [9] announced some results about generalized Sierpiński graphs concerning their automorphism groups and perfect codes. In our opinion, these results definitely deserve to be published. Later, the total chromatic number of generalized Sierpiński graphs was studied in [7] and the strong metric dimension has recently been studied in [5]. The authors of [33] obtained closed formulae for the chromatic, vertex cover, clique and domination numbers of generalized Sierpiński graphs $S(G, t)$ in terms of parameters of the base graph $G$. More recently, a general upper bound on the Roman domination number of $S(G, t)$ was obtained in [28]. In particular, it was studied the case in which the base graph $G$ is a path, a cycle, a complete graph or a graph having exactly one universal vertex. To the best of our knowledge, [34] is the first published paper studying the generalized Sierpiński graphs. In that article, the authors obtained closed formulae for the Randic index $R_{-1 / 2}$ of $S(G, t)$ and $P(G, t)$, where the base graph $G$ is a complete graph, a triangle-free regular graph or a bipartite semiregular graph. The present article is a continuation of [34] where we study the general Randić index $R_{\alpha}$ of Sierpiński-type polymeric networks. In particular, we 
obtain closed formulae for the general Randic index $R_{\alpha}$ of $S(G, t)$ and $P(G, t)$, where the base graph $G$, parameter $t$ and exponent $\alpha$ are arbitrary.

\section{Computing the General Randić index of $S(G, t)$}

From now on, given a graph $G=(V, E)$ and a specific edge $\{x, y\} \in E$, the number of copies of $\{x, y\}$ in $S(G, t)$, where vertex $x$ has degree $d(x)+l$ and vertex $y$ has degree $d(y)+l^{\prime}$ will be denoted by $f_{S(G, t)}\left(d(x)+l, d(y)+l^{\prime}\right)$, where $l, l^{\prime} \in\{0,1\}$. For instance, for the graph $S(G, 3)$ shown in Figure 2 we have $f_{S(G, 3)}(d(1), d(2))=21$ and $f_{S(G, 3)}(d(1)+1, d(2))=f_{S(G, 3)}(d(1), d(2)+1)=f_{S(G, 3)}(d(1)+1, d(2)+1)=$ 12. It can be noted that copies of a specific edge $\{x, y\} \in E$ in $S(G, t)$ are edges $\left\{w x y^{r}, w y x^{r}\right\}$, where $r \in\{0,1, \ldots, t-1\}$ and $w \in V^{t-1-r}$.

The set of neighbours that $x \in V$ has in $G$ will be denoted by $N(x)$, i.e.,

$$
N(x)=\{z \in V:\{x, z\} \in E\}
$$

Given two vertices $u, v \in V$, the number of triangles of $G$ containing $u$ and $v$ will be denoted by $\tau(u, v)$ and the number of triangles of $G$ will be denoted by $\tau(G) .{ }^{1}$

Note that $\sum_{\{u, v\} \in E(G)} \tau(u, v)=3 \tau(G)$. The complexity of counting the number of triangles of a graph $G$ is polynomial with respect to $|V|$. The trivial approach of counting the number of triangles is to check for every triple $x, y, z \in V$ if $x, y, z$ forms a triangle. This procedure gives us the algorithmic complexity of $O\left(n^{3}\right)$. However, this algorithmic complexity can be improved [3].

Notice that for any pair of adjacent vertices $u, v \in V$ we have $|N(u) \cap N(v)|=$ $\tau(u, v),|N(u) \cup N(v)|=d(u)+d(v)-\tau(u, v)$ and $|N(u)-N(v)|=d(u)-\tau(u, v)$. Given a graph of order $n$, from now on we will use the function $\psi(t)=1+n+n^{2}+\cdots+n^{t-1}=$ $\frac{n^{t}-1}{n-1}$.

Lemma 1. For any integer $t \geq 2$ and any edge $\{x, y\}$ of a graph $G$ of order $n$,

(i) $f_{S(G, t)}(d(x), d(y))=n^{t-2}(n-d(x)-d(y)+\tau(x, y))$.

(ii) $f_{S(G, t)}(d(x), d(y)+1)=n^{t-2}(d(y)-\tau(x, y))-\psi(t-2) d(x)$.

(iii) $f_{S(G, t)}(d(x)+1, d(y))=n^{t-2}(d(x)-\tau(x, y))-\psi(t-2) d(y)$.

(iv) $f_{S(G, t)}(d(x)+1, d(y)+1)=n^{t-2}(\tau(x, y)+1)+\psi(t-2)(d(x)+d(y)+1)$.

Proof. There are two different possibilities for the degree of any vertex $z x$ of $S(G, 2)$, namely $d(x)$ and $d(x)+1$, i.e., $z x$ has degree $d(x)+1$ for all $z \in N(x)$ and $z x$ has degree $d(x)$ for all $z \notin N(x)$. Therefore,

$$
f_{S(G, 2)}(d(x), d(y))=|V-(N(x) \cup N(y))|=n-d(x)-d(y)+\tau(x, y),
$$

\footnotetext{
${ }^{1} \mathrm{~A}$ triangle in a graph is a set of three vertices whose induced subgraph is isomorphic to $K_{3}$.
} 


$$
\begin{aligned}
& f_{S(G, 2)}(d(x), d(y)+1)=|N(y)-N(x)|=d(y)-\tau(x, y), \\
& f_{S(G, 2)}(d(x)+1, d(y))=|N(x)-N(y)|=d(x)-\tau(x, y), \\
& f_{S(G, 2)}(d(x)+1, d(y)+1)=|N(x) \cap N(y)|+1=\tau(x, y)+1
\end{aligned}
$$

For any $t \geq 3, w \in V^{t-1}$ and $z \in V$, the degree of $z w$ in $S(G, t)$ coincides with the degree of $w$ in $S(G, t-1)$, except when $w=j j \cdots j$ is an extreme vertex and $z \in N(j)$, in which case the degree of $z w=z j j \cdots j$ in $S(G, t)$ is $d(j)+1$ while the degree of $w=j j \cdots j$ in $S(G, t-1)$ is $d(j)$. Hence, we deduce the following:

$$
\begin{aligned}
\begin{aligned}
f_{S(G, t)}(d(x), d(y))= & n f_{S(G, t-1)}(d(x), d(y)) \\
= & n^{t-2}(n-d(x)-d(y)+\tau(x, y)), \\
f_{S(G, t)}(d(x), d(y)+1) & =n f_{S(G, t-1)}(d(x), d(y)+1)-d(x) \\
& =n^{t-2}(d(y)-\tau(x, y))-\sum_{i=0}^{t-3} n^{i} d(x) \\
& =n^{t-2}(d(y)-\tau(x, y))-\psi(t-2) d(x), \\
f_{S(G, t)}(d(x)+1, d(y)) & =n f_{S(G, t-1)}(d(x)+1, d(y))-d(y) \\
& =n^{t-2}(d(x)-\tau(x, y))-\sum_{i=0}^{t-3} n^{i} d(y) \\
& =n^{t-2}(d(x)-\tau(x, y))-\psi(t-2) d(y),
\end{aligned}
\end{aligned}
$$

and finally,

$$
\begin{aligned}
f_{S(G, t)}(d(x)+1, d(y)+1) & =n f_{S(G, t-1)}(d(x)+1, d(y)+1)+d(x)+d(y)+1 \\
& =n^{t-2}(\tau(x, y)+1)+\sum_{i=0}^{t-3} n^{i}(d(x)+d(y)+1) \\
& =n^{t-2}(\tau(x, y)+1)+\psi(t-2)(d(x)+d(y)+1) .
\end{aligned}
$$

Therefore, the result follows.

From the previous result we can deduce that the number of copies of $\{x, y\} \in E$ in $S(G, t)$ is $\sum_{l, l^{\prime} \in\{0,1\}} f_{S(G, t)}\left(d(x)+l, d(y)+l^{\prime}\right)=\psi(t)$. This result could also have been obtained by counting of these edges, considering the form of the words of vertices associated with them.

The main result of this section is Theorem 2 which provides a formula for the general Randic index of $S(G, t)$, where the base graph $G$ and $\alpha$ are arbitrary, and $t$ is an integer greater than one. 
Theorem 2. For any graph $G$ of order $n \geq 2$ and any integer $t \geq 2$,

$$
R_{\alpha}(S(G, t))=\sum_{\{x, y\} \in E} W_{\{x, y\}}
$$

where

$$
\begin{aligned}
W_{\{x, y\}} & =n^{t-2}(n-d(x)-d(y)+\tau(x, y)) d(x)^{\alpha} d(y)^{\alpha}+ \\
& +\left(n^{t-2}(d(y)-\tau(x, y))-\psi(t-2) d(x)\right) d(x)^{\alpha}(d(y)+1)^{\alpha}+ \\
& +\left(n^{t-2}(d(x)-\tau(x, y))-\psi(t-2) d(y)\right)(d(x)+1)^{\alpha} d(y)^{\alpha}+ \\
& +\left(n^{t-2}(\tau(x, y)+1)+\psi(t-2)(d(x)+d(y)+1)\right)(d(x)+1)^{\alpha}(d(y)+1)^{\alpha} .
\end{aligned}
$$

Proof. Since any copy of a vertex $z \in V$ in $S(G, t)$ has degree $d(z)$ or $d(z)+1$, the general Randic index of $S(G, t)$ can be expressed as

$$
R_{\alpha}(S(G, t))=\sum_{\{x, y\} \in E} \sum_{i=0}^{1} \sum_{j=0}^{1} f_{S(G, t)}(d(x)+i, d(y)+j)(d(x)+i)^{\alpha}(d(y)+j)^{\alpha} .
$$

Hence, by Lemma 1 the result immediately follows.

It can be noted that closed formulas can be obtained for several topological indices of $S(G, t)$ similarly to the previous proof. For instance, we are referring to the atombond connectivity index (ABC), geometric arithmetic index (GA), Harmonic index $(\mathrm{H})$, first Zagreb index $\left(M_{1}\right)$ and sum-connectivity index $(\chi)$.

The remaining results of this section are directly derived from Theorem 2 .

Corollary 3. For any $\delta$-regular graph $G$ of order $n$ and any integer $t \geq 2$,

$$
\begin{aligned}
R_{\alpha}(S(G, t)) & =\left(\frac{n^{t-1} \delta}{2}(n-2 \delta)+3 n^{t-2} \tau(G)\right) \delta^{2 \alpha} \\
& +\left(\left(n^{t-1}+\psi(t-1)\right) \delta^{2}-6 n^{t-2} \tau(G)\right) \delta^{\alpha}(\delta+1)^{\alpha} \\
& +\left(\frac{n \delta}{2} \psi(t-1)+n \delta^{2} \psi(t-2)+3 n^{t-2} \tau(G)\right)(\delta+1)^{2 \alpha}
\end{aligned}
$$

A complete graph $K_{n}$ of order $n \geq 2$ is $(n-1)$-regular and it has $\left(\begin{array}{l}n \\ 3\end{array}\right)$ triangles. Therefore, the next result follows.

Remark 4. For any integers $t, n \geq 2$,

$$
R_{\alpha}\left(S\left(K_{n}, t\right)\right)=n^{\alpha+1}(n-1)^{\alpha+1}+\frac{n^{2 \alpha+t+1}-2 n^{2(\alpha+1)}+n^{2 \alpha+1}}{2}
$$

Remark 5. For any integers $t \geq 2$ and $n \geq 4$,

$$
R_{\alpha}\left(S\left(C_{n}, t\right)\right)=4^{\alpha} n^{t-1}(n-4)+4 \cdot 6^{\alpha}\left(n^{t-1}-n \psi(t-2)\right)+9^{\alpha} n(\psi(t-1)+4 \psi(t-2)) .
$$


Corollary 6. Let $G=\left(U_{1} \cup U_{2}, E\right)$ be a bipartite $\left(\delta_{1}, \delta_{2}\right)$-semiregular graph of order $n=n_{1}+n_{2}$, where $\left|U_{1}\right|=n_{1}$ and $\left|U_{2}\right|=n_{2}$. Then for any integer $t \geq 2$,

$$
\begin{aligned}
R_{\alpha}(S(G, t)) & =n_{1} n^{t-2} \delta_{1}^{\alpha+1} \delta_{2}^{\alpha}\left(n-\delta_{1}-\delta_{2}\right)+n_{1} \delta_{1}^{\alpha+1}\left(\delta_{2}+1\right)^{\alpha}\left(\delta_{2} n^{t-2}-\delta_{1} \psi(t-2)\right) \\
& +n_{2}\left(\delta_{1}+1\right)^{\alpha} \delta_{2}^{\alpha+1}\left(\delta_{1} n^{t-2}-\delta_{2} \psi(t-2)\right) \\
& +n_{1} \delta_{1}\left(\delta_{1}+1\right)^{\alpha}\left(\delta_{2}+1\right)^{\alpha}\left(n^{t-2}+\left(\delta_{1}+\delta_{2}+1\right) \psi(t-2)\right) .
\end{aligned}
$$

Chemical trees are trees that have no vertex with degree greater than 4 . The graph $S\left(K_{1,3}, 2\right)$ is an example of a chemical tree. Notice that for any $t \geq 2$, the Sierpiński graph $S\left(K_{1,3}, t\right)$, is a chemical tree.

As a particular case of Corollary 6 we obtain the following result.

Remark 7. For any integers $r, t \geq 2$,

$$
\begin{aligned}
R_{\alpha}\left(S\left(K_{1, r}, t\right)\right) & =(r+1)^{\alpha}\left((r+1)^{t-1}(r-1)+1\right)+2^{\alpha} r^{\alpha+1}+ \\
& +(2(r+1))^{\alpha}\left(2(r+1)^{t-1}-r-2\right) .
\end{aligned}
$$

Corollary 8. Let $t, n$ be integers such that $t \geq 2$ and $n \geq 3$. Then

$$
R_{\alpha}\left(S\left(P_{2}, t\right)\right)=2^{\alpha+1}+\left(2^{t}-3\right) 2^{2 \alpha}
$$

and

$$
\begin{aligned}
R_{\alpha}\left(S\left(P_{n}, t\right)\right) & =2^{\alpha} n^{t-2}(n-3)\left(2^{\alpha} n-2^{\alpha+2}+2\right)+ \\
& +3^{\alpha}\left[2^{\alpha+2}(n-3)\left(n^{t-2}-\psi(t-2)\right)+4 n^{t-2}-2 \psi(t-2)\right]+ \\
& +2^{2 \alpha+1}\left(n^{t-2}-2 \psi(t-2)\right)+ \\
& +3^{\alpha}\left[3^{\alpha}(n-3)\left(n^{t-2}+5 \psi(t-2)\right)+2^{\alpha+1}\left(n^{t-2}+4 \psi(t-2)\right)\right] .
\end{aligned}
$$

In order to continue presenting our results, we need to introduce a definition. Given a graph $G$ on the vertex set $V$, we define the parameter

$$
M_{\alpha}(G)=\sum_{x \in V} d(x)^{\alpha}
$$

Note that $M_{1}(G)$ is equal to twice the number of edges of $G$ and $M_{2}(G)$ is the first Zagreb index. Considering that for any graph $G$ of maximum degree $\Delta(G)$ and minimum degree $\delta(G)$, and any vertex $x \in V(G)$ we have that $d(x)^{\alpha}+(\delta(G)+1)^{\alpha}-$ $\Delta(G)^{\alpha} \leq(d(x)+1)^{\alpha} \leq d(x)^{\alpha}+(\Delta(G)+1)^{\alpha}-\delta(G)^{\alpha}$ and replacing in Theorem 2, we deduce the following bounds.

Theorem 9. For any triangle free graph $G$ of order $n \geq 2$, maximum degree $\Delta$ and minimum degree $\delta$, and any integer $t \geq 2$,

$$
\beta_{L}(S(G, t)) \leq R_{\alpha}(S(G, t)) \leq \beta_{U}(S(G, t))
$$


where

$$
\begin{aligned}
\beta_{L}(S(G, t)) & =n^{t-2}(n-\Delta) R_{\alpha}(G)+ \\
& +2\left(n^{t-2} \delta-\Delta \psi(t-2)\right)\left(R_{\alpha}(G)+M_{\alpha+1}(G)\left[(\delta+1)^{\alpha}-\Delta^{\alpha}\right]\right)+ \\
& +\left(n^{t-2}+(2 \delta+1) \psi(t-2)\right)\left(R_{\alpha}(G)+2 M_{\alpha+1}(G)\left[(\delta+1)^{\alpha}-\Delta^{\alpha}\right]\right)+ \\
& +\left(n^{t-2}+(2 \delta+1) \psi(t-2)\right) \frac{M_{1}(G)}{2}\left[(\delta+1)^{\alpha}-\Delta^{\alpha}\right]^{2},
\end{aligned}
$$

and

$$
\begin{aligned}
\beta_{U}(S(G, t)) & =n^{t-2}(n-\delta) R_{\alpha}(G)+ \\
& +2\left(n^{t-2} \Delta-\delta \psi(t-2)\right)\left(R_{\alpha}(G)+M_{\alpha+1}(G)\left[(\Delta+1)^{\alpha}-\delta^{\alpha}\right]\right)+ \\
& +\left(n^{t-2}+(2 \Delta+1) \psi(t-2)\right)\left(R_{\alpha}(G)+2 M_{\alpha+1}(G)\left[(\Delta+1)^{\alpha}-\delta^{\alpha}\right]\right)+ \\
& +\left(n^{t-2}+(2 \Delta+1) \psi(t-2)\right) \frac{M_{1}(G)}{2}\left[(\Delta+1)^{\alpha}-\delta^{\alpha}\right]^{2} .
\end{aligned}
$$

Moreover, $\beta_{L}(S(G, t))=R_{\alpha}(S(G, t))=\beta_{U}(S(G, t))$ if and only if $G$ is a triangle free regular graph.

\section{Computing the General Randić index of $P(G, t)$}

The main result of this section is Theorem 14 which provides a formula for the general Randic index of $P(G, t)$, where the base graph $G$ and $\alpha$ are arbitrary, and $t$ is an integer greater than one.

From now on, given a graph $G$ and a vertex $x \in V(G)$, we refer to the degree of $x$ in $G$ as $d_{G}(x)$. If there is no ambiguity, we will continue writing $d(x)$.

Remark 10. For any graph $G$ of order $n \geq 2$,

$$
R_{\alpha}(P(G, 1))=n^{\alpha} \sum_{x \in V}(d(x)+1)^{\alpha}+\sum_{\{x, y\} \in E}(d(x)+1)^{\alpha}(d(y)+1)^{\alpha} .
$$

Proof. Note that the vertex $a_{1_{1}}$ has degree $n$ and it is adjacent to all vertices of $G$. Hence, for every vertex $x \in V$, there is one edge $\left\{a_{1_{1}}, x\right\}$, where $a_{1_{1}}$ has degree $n$ and $x$ has degree $d_{P(G, 1)}(x)=d_{G}(x)+1$. For the remaining edges $\{x, y\}$ we have that $d_{P(G, 1)}(x)=d_{G}(x)+1$ and $d_{P(G, 1)}(y)=d_{G}(y)+1$. Therefore, the result follows.

Corollary 11. For any $\delta$-regular graph $G$ of order $n \geq 2$,

$$
R_{\alpha}(P(G, 1))=n^{\alpha+1}(\delta+1)^{\alpha}+\frac{n \delta(\delta+1)^{2 \alpha}}{2} .
$$

In particular, for complete graphs,

$$
R_{\alpha}\left(P\left(K_{n}, 1\right)\right)=\frac{n^{2 \alpha+1}(n+1)}{2} .
$$


Corollary 12. Let $G=\left(U_{1} \cup U_{2}, E\right)$ be a bipartite $\left(\delta_{1}, \delta_{2}\right)$-semiregular graph of order $n=n_{1}+n_{2}$, where $\left|U_{1}\right|=n_{1}$ and $\left|U_{2}\right|=n_{2}$. Then,

$$
R_{\alpha}(P(G, 1))=n^{\alpha}\left(n_{1}\left(\delta_{1}+1\right)^{\alpha}+n_{2}\left(\delta_{2}+1\right)^{\alpha}\right)+n_{1} \delta_{1}\left(\left(\delta_{1}+1\right)\left(\delta_{2}+1\right)\right)^{\alpha} .
$$

Given a graph $G$ and a vertex $x \in V$, the number of copies of $x$ having degree $r$ in $S(G, t)$ will be denoted by $g_{S(G, t)}(r)$.

Lemma 13. For any graph $G$ of order $n$, any vertex $x \in V$ and any integer $t \geq 2$,

(i) $g_{S(G, t)}(d(x))=n^{t-1}-d(x) \psi(t-1)$.

(ii) $g_{S(G, t)}(d(x)+1)=d(x) \psi(t-1)$.

Proof. There are two different possibilities for the degree of any copy of vertex $x$ in $S(G, t)$, namely $d(x)$ and $d(x)+1$. Every copy of vertex $x$ having degree $d(x)+1$ in $S(G, t)$ is a vertex of the form $z_{1} \cdots z_{k} y x \cdots x$, where $y \in N(x), 0 \leq k \leq t-2$ and $z_{i} \in V(G)$ for $1 \leq i \leq k$. So,

$$
g_{S(G, t)}(d(x)+1)=d(x) \sum_{i=0}^{t-2} n^{i}=d(x) \psi(t-1) .
$$

On the other hand, every copy of vertex $x$ in $S(G, t)$ is a vertex of the form $z_{1} \cdots z_{t-1} x$, where $z_{i} \in V(G)$ for $1 \leq i \leq t-1$. Hence, we have $n^{t-1}$ copies of vertex $x$ in $S(G, t)$, and as a consequence,

$$
g_{S(G, t)}(d(x))=n^{t-1}-g_{S(G, t)}(d(x)+1)=n^{t-1}-d(x) \psi(t-1) .
$$

Now, we state a formula for the general Randic of $P(G, t)$, where the base graph $G$ and the exponent $\alpha$ are arbitrary, and $t$ is an integer greater than one.

Theorem 14. For any graph $G=(V, E)$ of order $n \geq 2$ and any integer $t \geq 2$,

$$
R_{\alpha}(P(G, t))=\sum_{i=1}^{7} \beta_{i}
$$

where

$$
\begin{aligned}
\beta_{1} & =n^{\alpha} \sum_{x \in V}(d(x)+2)^{\alpha}, \quad \beta_{2}=\sum_{\{x, y\} \in E}(d(x)+2)^{\alpha}(d(y)+2)^{\alpha}, \\
\beta_{3} & =(n+1)^{\alpha} n \psi(t-2) \sum_{x \in V}(d(x)+2)^{\alpha}+ \\
& +\frac{(n+1)^{\alpha}(t-2-n \psi(t-2))}{(n-1)} \sum_{x \in V} d(x)(d(x)+2)^{\alpha}+ \\
& +\frac{(n+1)^{\alpha}(t-2-n \psi(t-2))}{(1-n)} \sum_{x \in V} d(x)(d(x)+3)^{\alpha},
\end{aligned}
$$




$$
\begin{aligned}
& \beta_{4}=\sum_{\{x, y\} \in E} W_{4\{x, y\}} \text { with } \\
& W_{4\{x, y\}}=(d(x)+2)^{\alpha}(d(y)+2)^{\alpha}(n-d(x)-d(y)+\tau(x, y)) \psi(t-2)+ \\
& +(d(x)+2)^{\alpha}(d(y)+3)^{\alpha}\left((d(y)-\tau(x, y)) \psi(t-2)+d(x) \frac{t-2-\psi(t-2)}{n-1}\right)+ \\
& +(d(x)+3)^{\alpha}(d(y)+2)^{\alpha}\left((d(x)-\tau(x, y)) \psi(t-2)+d(y) \frac{t-2-\psi(t-2)}{n-1}\right)+ \\
& +(d(x)+3)^{\alpha}(d(y)+3)^{\alpha}(\tau(x, y)+1) \psi(t-2)+ \\
& +(d(x)+3)^{\alpha}(d(y)+3)^{\alpha}(d(x)+d(y)+1) \frac{t-2-\psi(t-2)}{1-n}, \\
& \beta_{5}=(n+1)^{\alpha} \psi(t-1) \sum_{x \in V}(d(x)+2)^{\alpha}+ \\
& +\frac{(n+1)^{\alpha}(t-1-\psi(t-1))}{(n-1)} \sum_{x \in V} d(x)(d(x)+2)^{\alpha}+ \\
& +\frac{(n+1)^{\alpha}(t-1-\psi(t-1))}{(1-n)} \sum_{x \in V} d(x)(d(x)+3)^{\alpha}, \\
& \beta_{6}=(n+1)^{\alpha} \sum_{x \in V}(d(x)+1)^{\alpha}\left(n^{t-1}-d(x) \psi(t-1)\right)+ \\
& +(n+1)^{\alpha} \psi(t-1) \sum_{x \in V} d(x)(d(x)+2)^{\alpha},
\end{aligned}
$$$$
\text { and } \beta_{7}=\sum_{\{x, y\} \in E} W_{7\{x, y\}} \text { with }
$$$$
\begin{aligned}
W_{7\{x, y\}} & =(d(x)+1)^{\alpha}(d(y)+1)^{\alpha} n^{t-2}(n-d(x)-d(y)+\tau(x, y))+ \\
& +(d(x)+1)^{\alpha}(d(y)+2)^{\alpha}\left(n^{t-2}(d(y)-\tau(x, y))-\psi(t-2) d(x)\right)+ \\
& +(d(x)+2)^{\alpha}(d(y)+1)^{\alpha}\left(n^{t-2}(d(x)-\tau(x, y))-\psi(t-2) d(y)\right)+ \\
& +(d(x)+2)^{\alpha}(d(y)+2)^{\alpha}\left(n^{t-2}(\tau(x, y)+1)+\psi(t-2)(d(x)+d(y)+1)\right) .
\end{aligned}
$$

Proof. Let $d(u), d(v)$ be degrees of $u, v$ in $P(G, t)$, respectively. We differentiate the following cases for any edge $\{u, v\}$ of $P(G, t)$.

1. $u=a_{1_{1}}$ and $v \in V_{1}$. In this case, there are $n$ edges $\{u, v\}$ with $d(u)=n$ and $d_{P(G, t)}(v)=d_{G}(v)+2$. Then the contribution of these edges to the General Randić index is equal to $\beta_{1}$.

2. $u, v \in V_{1}$. In this case, each extreme vertex of an edge $\{u, v\}$ has degree $d_{P(G, t)}(u)=d_{G}(u)+2$ and $d_{P(G, t)}(v)=d_{G}(v)+2$. So, the contribution of these edges to the General Randić index is equal to $\beta_{2}$. 
3. $u \in A_{i}$ and $v \in V_{i}$ for $2 \leq i \leq t-1$. We assume that $v=w x$, where $w \in V^{i-1}$ and $x \in V$. In this case $d(u)=n+1$ and, by Lemma 13, there are $g_{S(G, i)}(d(x)+1)=d(x) \psi(i-1)$ edges $\{u, v\}$ where $v$ has degree $d(v)=d(x)+3$ and there are $g_{S(G, i)}(d(x))=n^{i-1}-d(x) \psi(i-1)$ edges $\{u, v\}$ where $d(v)=$ $d(x)+2$. Thus, the contribution of these edges to the General Randić index is equal to $\sum_{i=2}^{t-1} \sum_{x \in V} W_{3\{x\}}^{\prime}$, where,

$$
W_{3\{x\}}^{\prime}=(n+1)^{\alpha} \sum_{i=0}^{1}(d(x)+i+2)^{\alpha} g_{S(G, i)}(d(x)+i) .
$$

Since $\sum_{i=2}^{t-1} n^{i-1}=n \psi(t-2)$ and $\sum_{i=2}^{t-1} \psi(i-1)=\frac{t-2-n \psi(t-2)}{1-n}$, we obtain $\beta_{3}=\sum_{i=2}^{t-1} \sum_{x \in V} W_{3\{x\}}^{\prime}$

4. $u, v \in V_{i}$, for $2 \leq i \leq t-1$. We assume that $u=w x$ and $v=w^{\prime} y$, where $w, w^{\prime} \in V^{i-1}$ and $\{x, y\} \in E$. By Lemma 1 , there are

$f_{S(G, i)}(d(x), d(y))=n^{i-2}(n-d(x)-d(y)+\tau(x, y))$ edges $\{u, v\}$ where $d(u)=$ $d(x)+2$ and $d(v)=d(y)+2$,

$f_{S(G, i)}(d(x), d(y)+1)=n^{i-2}(d(y)-\tau(x, y))-\psi(i-2) d(x)$ edges $\{u, v\}$ where $d(u)=d(x)+2$ and $d(v)=d(y)+3$,

$f_{S(G, i)}(d(x)+1, d(y))=n^{i-2}(d(x)-\tau(x, y))-\psi(i-2) d(y)$ edges $\{u, v\}$ where $d(u)=d(x)+3$ and $d(v)=d(y)+2$,

$f_{S(G, i)}(d(x)+1, d(y)+1)=n^{i-2}(\tau(x, y)+1)+\psi(i-2)(d(x)+d(y)+1)$ edges $\{u, v\}$ where $d(u)=d(x)+3$ and $d(v)=d(y)+3$.

Hence, the contribution of these edges to the General Randic index is equal to $\sum_{i=2}^{t-1} \sum_{\{x, y\} \in E} W_{4\{x, y\}}^{\prime}$, where,

$$
W_{4\{x, y\}}^{\prime}=\sum_{i=0}^{1} \sum_{j=0}^{1}(d(x)+i+2)^{\alpha}(d(y)+j+2)^{\alpha} f_{S(G, i)}(d(x)+i, d(y)+j) .
$$

Since $\sum_{i=2}^{t-1} n^{i-2}=\psi(t-2)$ and $\sum_{i=2}^{t-1} \psi(i-2)=\frac{t-2-\psi(t-2)}{1-n}$, we obtain $\beta_{4}=$ $\sum_{i=2}^{t-1} \sum_{\{x, y\} \in E} W_{4\{x, y\}}^{\prime}=\sum_{\{x, y\} \in E} W_{4\{x, y\}}$. 
5. $u \in A_{i+1}$ and $v \in V_{i}$ for $1 \leq i \leq t-1$. We assume that $v=w x$, where $w \in V^{i-1}$ and $x \in V$. In this case $d(u)=n+1$ and, by Lemma 13, there are $g_{S(G, i)}(d(x)+1)=d(x) \psi(i-1)$ edges $\{u, v\}$ where $v$ has degree $d(v)=d(x)+3$ and there are $g_{S(G, i)}(d(x))=n^{i-1}-d(x) \psi(i-1)$ edges $\{u, v\}$ where $d(v)=$ $d(x)+2$. Hence, the contribution of these edges to the General Randic index is equal to $\sum_{i=2}^{t-1} \sum_{x \in V} W_{5\{x\}}^{\prime}$, where,

$$
W_{5\{x\}}^{\prime}=(n+1)^{\alpha} \sum_{i=0}^{1}(d(x)+i+2)^{\alpha} g_{S(G, i)}(d(x)+i) .
$$

Since $\sum_{i=1}^{t-1} n^{i-1}=\psi(t-1)$ and $\sum_{i=1}^{t-1} \psi(i-1)=\frac{t-1-n \psi(t-1)}{1-n}$, we obtain $\beta_{5}=\sum_{i=2}^{t-1} \sum_{x \in V} W_{5\{x\}}^{\prime}$

6. $u \in A_{t}$ and $v \in V_{t}$. We assume that $v=w x$, where $w \in V^{t-1}$ and $x \in V$. As above $d(u)=n+1$ and, by Lemma 13, there are $g_{S(G, t)}(d(x)+1)=d(x) \psi(t-1)$ edges $\{u, v\}$ where $v$ has degree $d(v)=d(x)+2$ and there are $g_{S(G, t)}(d(x))=$ $n^{t-1}-d(x) \psi(t-1)$ edges $\{u, v\}$ where $d(v)=d(x)+1$. Thus, the contribution of these edges to the General Randić index is equal to $\beta_{6}$.

7. $u, v \in V_{t}$. We assume that $u=w x$ and $v=w^{\prime} y$, where $w, w^{\prime} \in V^{t-1}$ and $x, y \in V$. By Lemma 1, there are

$$
\begin{aligned}
& f_{S(G, t)}(d(x), d(y))=n^{t-2}(n-d(x)-d(y)+\tau(x, y)) \text { edges }\{u, v\} \text { where } d(u)= \\
& d(x)+1 \text { and } d(v)=d(y)+1, \\
& f_{S(G, t)}(d(x), d(y)+1)=n^{t-2}(d(y)-\tau(x, y))-\psi(t-2) d(x) \text { edges }\{u, v\} \text { where } \\
& d(u)=d(x)+1 \text { and } d(v)=d(y)+2, \\
& f_{S(G, t)}(d(x)+1, d(y))=n^{t-2}(d(x)-\tau(x, y))-\psi(t-2) d(y) \text { edges }\{u, v\} \text { where } \\
& \quad d(u)=d(x)+2 \text { and } d(v)=d(y)+1, \\
& f_{S(G, t)}(d(x)+1, d(y)+1)=n^{t-2}(\tau(x, y)+1)+\psi(t-2)(d(x)+d(y)+1) \text { edges } \\
& \{u, v\} \text { where } d(u)=d(x)+2 \text { and } d(v)=d(y)+2 .
\end{aligned}
$$

Hence, the contribution of these edges to the General Randic index is equal to $\beta_{7}=\sum_{\{x, y\} \in E} W_{7\{x, y\}}$

According to the seven cases above, the result follows.

Now, we will show some particular cases of Theorem 14 . 
Corollary 15. For any $\delta$-regular graph $G$ of order $n \geq 2$ and any integer $t \geq 2$,

$$
R_{\alpha}(P(G, t))=\sum_{i=1}^{7} \beta_{i}
$$

where

$$
\begin{aligned}
\beta_{1} & =n^{\alpha+1}(\delta+2)^{\alpha}, \quad \beta_{2}=\frac{n \delta(\delta+2)^{2 \alpha}}{2}, \\
\beta_{3} & =n^{2} \psi(t-2)(n+1)^{\alpha}(\delta+2)^{\alpha}+ \\
& +\frac{n \delta(n+1)^{\alpha}(\delta+2)^{\alpha}(t-2-n \psi(t-2))}{(n-1)}+ \\
& +\frac{n \delta(n+1)^{\alpha}(\delta+3)^{\alpha}(t-2-n \psi(t-2))}{(1-n)}, \\
\beta_{4} & =(\delta+2)^{2 \alpha} \psi(t-2)\left(\frac{n \delta(n-2 \delta)}{2}+3 \tau(G)\right)+ \\
& +(\delta+2)^{\alpha}(\delta+3)^{\alpha}\left(\left(n \delta^{2}-6 \tau(G)\right) \psi(t-2)+\frac{n \delta^{2}(t-2-\psi(t-2))}{n-1}\right)+ \\
& +(\delta+3)^{2 \alpha}\left(\left(3 \tau(G)+\frac{n \delta}{2}\right) \psi(t-2)+\frac{n \delta(2 \delta+1)(t-2-\psi(t-2))}{2(1-n)}\right), \\
\beta_{5} & =\frac{n(n+1)^{\alpha}(\delta+2)^{\alpha}(\delta(t-1)+\psi(t-1)(n-\delta-1))}{(n-1)}+ \\
& +\frac{n \delta(n+1)^{\alpha}(\delta+3)^{\alpha}(t-1-\psi(t-1))}{(1-n)},
\end{aligned}
$$$$
\beta_{6}=(n+1)^{\alpha}(\delta+1)^{\alpha}\left(n^{t}-n \delta \psi(t-1)\right)+n \delta(n+1)^{\alpha}(\delta+2)^{\alpha} \psi(t-1),
$$

and

$$
\begin{aligned}
\beta_{7} & =(\delta+1)^{2 \alpha} n^{t-2}\left(\frac{n \delta}{2}(n-2 \delta)+3 \tau(G)\right)+ \\
& +(\delta+1)^{\alpha}(\delta+2)^{\alpha}\left(\delta^{2}\left(n^{t-1}-n \psi(t-2)\right)-6 n^{t-2} \tau(G)\right)+ \\
& +(\delta+2)^{2 \alpha}\left(\frac{n \delta}{2} \psi(t-1)+n \delta^{2} \psi(t-2)+3 n^{t-2} \tau(G)\right) .
\end{aligned}
$$

As we mentioned above, a complete graph $K_{n}$ of order $n \geq 2$ is $(n-1)$-regular and it has $\left(\begin{array}{l}n \\ 3\end{array}\right)$ triangles. Therefore, the next result is deduced from Corollary 15. 
Corollary 16. For any integers $n, t \geq 2$,

$$
R_{\alpha}\left(P\left(K_{n}, t\right)\right)=\sum_{l=1}^{7} \beta_{l},
$$

where

$$
\begin{aligned}
& \beta_{1}=n^{\alpha+1}(n+1)^{\alpha}, \quad \beta_{2}=\frac{n(n-1)(n+1)^{2 \alpha}}{2}, \\
& \beta_{3}=n(t-2)(n+1)^{2 \alpha}+n(n+1)^{\alpha}(n+2)^{\alpha}(2+n \psi(t-2)-t), \\
& \beta_{4}=(t-2) n(n-1)(n+1)^{\alpha}(n+2)^{\alpha}+\frac{(n+2)^{2 \alpha}}{2}\left(n^{3} \psi(t-2)+(t-2)\left(n-2 n^{2}\right)\right), \\
& \beta_{5}=(t-1) n(n+1)^{2 \alpha}+n(n+1)^{\alpha}(n+2)^{\alpha}(\psi(t-1)-(t-1)), \\
& \beta_{6}=n^{\alpha+1}(n+1)^{\alpha}+\left(n^{t}-n\right)(n+1)^{2 \alpha}, \\
& \beta_{7}=(n-1) n^{\alpha+1}(n+1)^{\alpha}+\frac{\left(n^{t+1}-2 n^{2}+n\right)(n+1)^{2 \alpha}}{2} .
\end{aligned}
$$

\section{References}

[1] A. Blumen, A. Jurjiu, Multifractal spectra and the relaxation of model polymer networks, The Journal of Chemical Physics 116 (6) (2002) 2636-2641. doi: $10.1063 / 1.1433744$

[2] K. V. Camarda, C. D. Maranas, Optimization in polymer design using connectivity indices, Industrial \& Engineering Chemistry Research 38 (5) (1999) 1884-1892.doi:10.1021/ie980682n

[3] N. Chiba, T. Nishizeki, Arboricity and subgraph listing algorithms, SIAM Journal on Computing 14 (1) (1985) 210-223. doi:10.1137/0214017

[4] K. C. Das, I. Gutman, Some properties of the second Zagreb index, MATCH Communications in Mathematical and in Computer Chemistry (52) (2004) 103-112.

URL http://match.pmf.kg.ac.rs/electronic_versions/Match52/match52_103-112.pdf

[5] E. Estaji, J. A. Rodríguez-Velázquez, The strong metric dimension of generalized Sierpiński graphs with pendant vertices, Ars Mathematica Contemporanea 12 (1) (2017) 127-134.

URL http://amc-journal.eu/index.php/amc/article/view/813 
[6] J. Gao, M. Lu, On the Randić index of unicyclic graphs, MATCH Communications in Mathematical and in Computer Chemistry 53 (2) (2005) 377-384.

URL http://match.pmf.kg.ac.rs/electronic_versions/Match53/n2/match53n2_377-384.p

[7] J. Geetha, K. Somasundaram, Total coloring of generalized Sierpiński graphs, The Australasian Journal of Combinatorics 63 (2015) 58-69.

URL https://ajc.maths.uq.edu.au/pdf/63/ajc_v63_p058.pdf

[8] S. Gravier, M. Kovše, M. Mollard, J. Moncel, A. Parreau, New results on variants of covering codes in Sierpiński graphs, Designs, Codes and Cryptography 69 (2) (2013) 181-188. doi:10.1007/s10623-012-9642-1

[9] S. Gravier, M. Kovše, A. Parreau, Generalized Sierpiński graphs, EuroComb 2011 (Poster), Rényi Institute, Budapest, 2011.

URL http://www.renyi.hu/conferences/ec11/posters/parreau.pdf

[10] I. Gutman, B. Furtula (eds.), Recent Results in the Theory of Randić Index in MATHEMATICAL CHEMISTRY MONOGRAPHS, No. 6, University of Kragujevac and Faculty of Science Kragujevac, 2008.

URL http://match.pmf . kg.ac.rs/mcm6.htm

[11] I. Gutman, M. Lepović, Choosing the exponent in the definition of the connectivity index, Journal of the Serbian Chemical Society 66 (9) (2001) 605-611.

URL http://www.shd.org.rs/JSCS/Vo166/No9/V66-No9-05.pdf

[12] A.M. Hinz, S. Klavžar, S.S. Zemljič, A survey and classification of Sierpiński-type graphs, Discrete Applied Mathematics 217 (2017) 565-600. doi:10.1016/j.dam.2016.09.024

[13] A. Hinz, D. Parisse, The Average Eccentricity of Sierpiński Graphs, Graphs and Combinatorics 28 (5) (2012) 671-686. doi:10.1007/s00373-011-1076-4

[14] A. M. Hinz, C. H. auf der Heide, An efficient algorithm to determine all shortest paths in Sierpiński graphs, Discrete Applied Mathematics 177 (2014) 111-120. doi:10.1016/j.dam.2014.05.049

[15] Y. Hu, X. Li, Y. Shi, T. Xu, I. Gutman, On molecular graphs with smallest and greatest zeroth-order general Randić index, MATCH Communications in Mathematical and in Computer Chemistry 54 (2) (2055) 425-434.

URL http://match.pmf.kg.ac.rs/electronic_versions/Match54/n2/match54n2_425-434.pc

[16] A. Jurjiu, T. Koslowski, A. Blumen, Dynamics of deterministic fractal polymer networks: Hydrodynamic interactions and the absence of scaling, The Journal of Chemical Physics 118 (5) (2003) 2398-2404. doi:10.1063/1.1534576

[17] A. G. Jurjiu, Dynamics of polymer networks modelled by finite regular fractals, Ph.D. thesis, Fakultät für Mathematik und Physik, Albert-Ludwigs-Universität Freiburg im Breisgau (2005).

URL https://www.freidok. uni-freiburg.de/fedora/objects/freidok:2734/datastreams/ 
[18] S. Klavžar, U. Milutinović, Graphs $S(n, k)$ and a variant of the Tower of Hanoi problem, Czechoslovak Mathematical Journal 47 (1) (1997) 95-104.

URL http://dml.cz/dmlcz/127341

[19] S. Klavžar, U. Milutinović, C. Petr, 1-perfect codes in Sierpiński graphs, Bulletin of the Australian Mathematical Society 66 (3) (2002) 369-384. doi:10.1017/S0004972700040235

[20] S. Klavžar, I. Peterin, S. S. Zemljič, Hamming dimension of a graph-the case of Sierpiński graphs, European Journal of Combinatorics 34 (2) (2013) 460-473. doi:10.1016/j.ejc.2012.09.006

[21] S. Klavžar, S. S. Zemljič, On distances in Sierpiński graphs: Almost-extreme vertices and metric dimension, Applicable Analysis and Discrete Mathematics 7 (1) (2013) 72-82. doi:10.2298/AADM130109001K

[22] H. Li, M. Lu, The $m$-connectivity index of graphs, MATCH Communications in Mathematical and in Computer Chemistry 54 (2) (2005) 417-423.

URL http://match.pmf .kg.ac.rs/electronic_versions/Match54/n2/match54n2_417-423.pc

[23] X. Li, I. Gutman, Mathematical Aspects of Randić-type Molecular Structure Descriptors, Mathematical Chemistry Monographs, No. 1, University of Kragujevac and Faculty of Science Kragujevac, 2006.

URL http://match.pmf .kg.ac.rs/mcm1.htm

[24] X. Li, Y. Shi, A survey on the Randić index, MATCH Communications in Mathematical and in Computer Chemistry 59 (1) (2008) 127-156.

URL http://match.pmf .kg.ac.rs/electronic_versions/Match59/n1/match59n1_127-156.pc

[25] B. Liu, I. Gutman, On general Randić indices, MATCH Communications in Mathematical and in Computer Chemistry 58 (1) (2007) 147-154. URL http://match.pmf .kg.ac.rs/electronic_versions/Match58/n1/match58n1_147-154.pc

[26] J. Liu, The Randić index and girth of triangle-free graphs, Ars Combinatoria 113 (2014) 289-297.

URL http://www. combinatorialmath.ca/arscombinatoria/vol113.html

[27] D. Parisse, On some metric properties of the Sierpiński graphs $S(n, k)$, Ars Combinatoria 90 (2009) 145-160.

URL http://www . combinatorialmath.ca/arscombinatoria/vol90.html

[28] F. Ramezani, E. D. Rodriguez-Bazan, J. A. Rodríguez-Velázquez, On the Roman domination number of generalized Sierpiński graphs, Filomat, to appear. URL https://arxiv.org/pdf/1605.06918.pdf

[29] M. Randić, On characterization of molecular branching, Journal of the American Chemical Society 97 (23) (1975) 6609-6615. doi:10.1021/ja00856a001 
[30] M. Randić, On history of the Randić index and emerging hostility toward chemical graph theory, MATCH Communications in Mathematical and in Computer Chemistry 59 (1) (2008) 5-124.

URL http://match.pmf.kg.ac.rs/content59n1.htm

[31] J. A. Rodríguez, A spectral approach to the Randić index, Linear Algebra and its Applications 400 (2005) 339-344. doi:10.1016/j.laa.2005.01.003

[32] J. A. Rodríguez, J. M. Sigarreta, On the Randić index and conditional parameters of a graph, MATCH Communications in Mathematical and in Computer Chemistry 54 (2) (2005) 403-416.

URL http://match.pmf.kg.ac.rs/electronic_versions/Match54/n2/match54n2_403-416.pc

[33] J. A. Rodríguez-Velázquez, E. D. Rodríguez-Bazan, A. Estrada-Moreno, On Generalized Sierpiński Graphs, Discussiones Mathematicae Graph Theory 37 (3) (2017) 547-560. doi:10.7151/dmgt.1945

[34] J. A. Rodríguez-Velázquez, J. Tomás-Andreu, On the Randić index of polymer networks modelled by generalized Sierpiński graphs, MATCH Communications in Mathematical and in Computer Chemistry 74 (1) (2015) 145-160.

URL http://match.pmf .kg.ac.rs/electronic_versions/Match74/n1/match74n1_145-160.pc

[35] R. Stepto (ed.), Polymer Networks. Principles of their Formation, Structure and Properties, Springer Netherlands, 1998.

URL http://www. springer.com/in/book/9780751402643

[36] R. Todeschini, V. Consonni, Handbook of Molecular Descriptors, Methods and Principles in Medicinal Chemistry, WILEY-VCH Verlag GmbH, 2008. doi:10.1002/9783527613106

[37] W. Wang, X. Hou, W. Ning, The $k$-connectivity index of an infinite class of dendrimer nanostars, Digest Journal of Nanomaterials and Biostructures 6 (3) (2011) 1199-1205.

URL http://www. chalcogen.ro/1199_Wang.pdf

[38] I. G. Yero, J. A. Rodríguez-Velázquez, I. Gutman, Estimating the higherorder Randić index, Chemical Physics Letters 489 (1-3) (2010) 118-120. doi:10.1016/j.cplett.2010.02.052 